\title{
The Status Quo and Trend of Artificial Intelligence in the Field of Education in China and Russia
}

\author{
Li Baohong \\ Department of Teacher Education, Lomonosov Moscow State University, Moscow, Russia
}

\section{Email address:}

libaohong9785@163.com

\section{To cite this article:}

Li Baohong. The Status Quo and Trend of Artificial Intelligence in the Field of Education in China and Russia. Science Innovation. Vol. 7, No. 4, 2019, pp. 133-136. doi: 10.11648/j.si.20190704.16

Received: November 3, 2019; Accepted: November 25, 2019; Published: December 3, 2019

\begin{abstract}
With the advent of 5G, the research and development of modern educational information technology has once again attracted the attention of the education field, in which artificial intelligence has become the key word for innovative research. In recent years, China and Russia have maintained close ties in various fields, especially the establishment of the "New Strategic Cooperation Partnership between China and Russia in the New Era", which has pushed Sino-Russian relations to a climax. In a period of great historical significance. How to combine the characteristics of the two countries in the field of education, complement each other's advantages, achieve cooperation and win-win, and promote the development of science and technology in education is our common goal. By analyzing and comparing the development status and characteristics of artificial intelligence in the two countries, it summarizes the potential, prospects and constructive opinions on the common development of China and Russia.
\end{abstract}

Keywords: Education, Artificial Intelligence, Status and Trends

\section{人工智能在中俄两国教育领域发展现状及趋势}

\section{李保宏}

莫斯科国立罗蒙诺索夫大学教育系, 莫斯科, 俄罗斯

\section{邮箱}

libaohong9785@163.com

摘要：随着 $5 \mathrm{G}$ 的问世, 现代教育信息技术的研究与发展再一次引起教育领域的关注, 其中人工智能成为了创新科研 的关键词。近年来, 中俄两国在各领域联系密切, 特别是“新时代中俄全面战略协作伙伴关系”的确立, 将中俄关系推 向了高潮。在具有重大历史意义的时期。如何将两国在教育领域的特点相结合, 优势互补, 达到合作共赢, 从而推动 教育科技化发展, 是我们共同的目标。通过分析和对比两国人工智能的发展现状及特点, 总结提出中俄双方共同发展 具有的潜力、前景和关于合作的建设性意见。

关键词：教育，人工智能，发展与趋势

\section{1. 引言}

新时代背景下, 人工智能一词作为信息文化的一部分 早已不陌生, 从该概念出现至今的 60 多年来, 已经迎来了
发展的第三次浪潮。世界各国都为掌握和应用人工智能技 术投入大量的人力物力。人工智能产品也涉及到各个行业, 它的迅速发展, 必将改变一代人甚至多代人的生活和生产 方式。在教育这一重要领域扮演的角色更是不容忽视。中 
国和俄罗斯作为世界大国，在现代教育信息技术方面以自 身优势为基础, 取长补短, 相互支持, 是合作互赢的关键。

\section{2. 人工智能的概述}

人工智能 (Artificial Intelligence, 缩写为 $\mathrm{AI}$ ) 是数字 计算机或计算机控制的机器人执行通常与智能生物有关 的任务的能力。简而言之, AI是大脑中神经元的粗略显示。 信号从神经元传递到神经元, 最后输出-获得数值, 分类 或生成结果。人类为了研发出智能机器和系统作为助手, 几十年来不断探索和创新, 终于在除软件以外的, 教育科 研、网络、金融和制造业等领域有所突破, 取得了不错的 阶段性成果。其中最引起关注的就是在教育领域的发展与 应用。[11]

\section{3. 人工智能在中国教育领域的发展现状与应用}

\section{1. 人工智能在中国教育领域中的研究现状}

随着 $5 \mathrm{G}$ 时代的到来, 人工智能越来越影响我们的生活, 在我国教育领域中的应用可以概括为五个方面:

人工智能使学习环境越来越智能化。例如智慧教室、 智慧图书馆、智慧书写系统以及校园安全预警系统等。利 用智能的感知和管控使学生的学习环境更加便捷和安全。

人工智能使学习过程越来越智能化。在学习过程中人 工智能可以提供前所未有的技术支持, 可以通过数据对学 生的知识结构、能力结构进行表达, 便于教师及时掌握学 生学习情况。人工智能还可以根据实际需求进行资源推荐。 同时也可以检测学生学习的状态, 如兴奋、疲劳等。除此 之外, 人工智能可以结合学习工具VR和 $A R$ 进入特定的虚 拟环境, 适用于学习使用过于复杂, 危险和昂贵的教学系 统和流程。[5]

人工智能可以对学习过程的评价起到重要作用。它可 以掌握学生在学习过程中对各个知识点的掌握情况, 甚至 可以掌握学生的心里素养和体质发展情况。可以使教学评 价由单一评价转变为客观综合评价。[3]

人工智能可以辅助教师的工作, 成为“助手”。根据学 生的学习和平时表现为学生制定个性化服务, 做出独特的 评价, 减轻教师的工作, 这也正符合“中国教育现代化 2035 ” 要推进兼有个性化和规模化教育的要求。[4]

人工智能可以在教育管理、教育决策起到重要作用。 通过网络提供教育个性化服务, 打破了以往传统的工作模 式, 例如老师在线提供教学以及针对学生不同问题进行解 答。

\section{2. 人工智能在中国教育领域中的应用实例}

\subsection{1. 海风教育AI系统好望角}

国内在线教育领域首个落地 $\mathrm{AI}$ 应用成果, 适用于线上 教育, 通过对人脸面部表情的识别, 分析学生的情绪, 判 断学生的专注力, 及时将结果反馈给教师, 便于及时调整 教学方式和进度, 2018年4月正式推出, 旨在教学不受地 域控制, 减轻老师工作量, 提高教学质量。在线英语教学
VIPKID采用相似的方式, 融合人脸技术, 收集上课数据, 实时判断学生情绪, 将评价结果反馈给老师。[13]

\subsection{2. 在线学习平台MOOC慕课}

该平台进入中国后迅速得到了学生的青睐, 注册用户 由 2013 年的 13 万人到目前的超过 7000 万人, 足以说明这种 创新型教学方式取得了不错的成绩。它不同于传统的一对 一或一对多教学, 而是集结了来自世界各地有共同问题或 同一主题学习诉求的学生, 整合成一个小团体进行讲解和 讨论。主要特点是规模大、开放性和网络课程。满足了非 全日制学生, 在职进修, 学习第二专业等需求。是国际上 比较成熟的线上学习平台。

\subsection{3. 杭州中学“智慧课堂行为管理系统”}

杭州第十一中学在2018年5月开始试行的“智慧课堂 行为管理系统” 是人工智能技术在教育领域应用的典型示 例。该系统在传统的一对多教学模式上通过安装摄像头, 在不涉及隐私的情况下采集学生上课时的表情和动作信 息, 对学生的专注度进行打分, 将判定结果反馈给教师, 便于根据课堂状态及时做出调整, 从而促进教学改进。[12]

\subsubsection{CCS课堂呵护系统}

由汉王教育公司推出的CCS课堂呵护系统同样适用 于线下教学。与“智慧课堂行为管理系统”不同的是, 该系 统在捕捉和分析学生学习状态之后, 将测评结果根据关注 点不同形成不同版本的报告发送到校长, 教师和家长三个 终端。便于学校从整体, 教师从全班, 家长从个体角度出 发, 及时了解所需关键信息。该方法可以大致了解学生的 状态和上课的活跃度, 起到教学辅助作用, 不能以此为标 准判断学生是否好好学习。[7]

\section{4. 人工智能在俄罗斯教育领域的发展现状与应 用}

\section{1. 人工智能在俄罗斯教育领域中的发展现状}

新华网在2018年9月9日发布了人工智能技术在全球 普及度。美国、中国和印度位列前三, 榜单中的 22 个国家 中未见俄罗斯的身影。[14]这确实反映出目前俄罗斯在人 工智能方面发展缓慢, 但俄罗斯的学者们并没有就此放弃。 莫斯科国立罗蒙诺索夫大学哲学系教师埃琳娜. 布兹加琳 娜（Elena Bryzgalina）在2019年8月21日发表了题为“教育 中的人工智能: 社会哲学层面”的文章。多角度归纳了人 工智能在教学环节中的应用以及面临的问题, 并举美国, 中国和英国的具体实例加以说明。[9]

莫斯科国立大学校长V.A.萨多夫尼西院士在 2019 年 3 月 25 日开幕的题为 “大学, 社会与人类未来”国际论坛上发 表演讲时说到“人工智能可以掌握数字空间, 并在其中填 充可靠的信息, 这是科学的最新成果。今天, 我们需要一 个电子教育环境, 因为我们还必须掌握数字空间”。[15]

这也表示以莫大为首的具有高水平的俄罗斯计算机 将把人工智能研发提上日程。 
就在不久前的 10 月, 俄罗斯联邦总统普京签署了俄罗 斯联邦2030年前国家人工智能发展战略。该战略从人工智 能在俄罗斯和世界的发展, 人工智能技术发展与应用的基 本原则, 人工智能技术发展与应用的优先方向, 人工智能 发展目标和主要任务以及该战略的实施机制五个方面规 划了未来十年俄罗斯在人工智能领域的发展方向和任务。 其中明确的指出了在应用的优先方向就包含教育领域。 [16]为了达到提高教育领域服务质量的目标, 需要从多个 角度出发进行规划, 包括: 教学过程与学生及劳动力市场 需求相适应, 系统分析教育有效性指标, 从而优化职业导 向, 及早发现具有杰出能力的学生、自动化进行学业质量 评估和学习效果分析等。

\section{2. 人工智能在俄罗斯教育领域中的应用}

前面说到俄罗斯在人工智能方面起步相对较晚, 具体 的人工智能产品在教育领域的应用还相对较少。据俄媒报 道, 在俄罗斯西部城市彼尔姆的一座学校2019年初已经开 始尝试利用人工智能技术分析学生在校时的状态, 一方面 通过对学生面部表情的捕捉, 判断出七种情绪。当有学生 表现出负面的情绪时, 系统会将分析结果向老师报告。从 而使人类老师更加关注该学生, 避免发生负面的、伤害或 损害其他人的状况发生。另一方面, 如果系统运行状况乐 观, 将考虑应用到学生高考的考场上, 以便实时检测学生 考试状态。[10]在该产品的试用阶段, 虽获得了大部分家 长的许可, 但仍有少部分家长坚决反对使用这种信息技术 对孩子进行分析, 他们认为, 孩子在成长过程中应有自己 的隐私, 要顺应其自然生长。虽然产品在技术层面可以帮 助教师从事教学和管理工作, 但在情感方面仍然面临一些 困难需要克服。所以在学校普及使用新技术产品仍有大量 周边工作需要完成。

人工智能技术在俄罗斯最初应用到金融领域, 如最大 的银行Sberbank。这家银行投资建立了一所“21世纪学校”, 旨在培养信息技术领域的专家。学校课程主要涉及人工智 能、编程、算法、移动开发等方面。当前, 学校的注册学 生为 940 人, 平均学习时间为 $2 \sim 3$ 年或 5 年, 课程包括两次 为期各 6 个月的公司实习。[1]这所学校得到了俄罗斯联邦 总统普京的高度重视, 曾在2019年5月30日对该校进行了 访问并讲话。他表示: 人工智能发展水平已经成为全球竞 争的重要指标, 世界上众多发达国家都已经颁布了该领域 的行动纲领。俄罗斯政府同样颁布全面发展人工智能技术 的战略性文件以确保技术主权。普京明确的态度表现了对 本国在人工智能方面发展的信心和决心。[8]当结合自身优 势找到研发突破口后, 俄罗斯在信息技术方面定会发生巨 大变革。

\section{5. 中俄两国在教育信息技术方面各自优势及合 作建议}

自从2018年发布《教育信息化2.0行动计划》中国教 育信息化从量变阶段, 强调应用驱动、融合发展的 1.0 时 代正式迈进注重引领创新、生态变革的质变阶段。[2]我国 在教育信息技术方面的优势在于已经建设并全面推进基
础设施平台, 具有大规模的应用场景, 中国市场的残酷竞 争能使技术项目快速落地。大规模、数量多的互联网使用 方便收集大数据。[6]

俄罗斯人工智能领域具有广阔的前景, 原因不仅在于 俄罗斯互联网和移动通信技术高速发展, 其网络访问成本 较低, 还依托于高水平的数学、物理学科优势, 俄罗斯学 生团队已连续 8 年在世界编程锦标赛上获得奖项。俄罗斯 具备在人工智能领域不断创新和开发、向世界水平靠拢的 条件。[1]

综上所述, 中俄两国既是世界大国, 又是友好邻邦, 在人工智能方面具有不同的优势。在中俄建交 70 年的今天, 为推动两国在教育领域人工智能技术的优势互补试做出 以下建议: 1. 在政府的支持下, 具有优势学课的高校增设 人工智能研究专业或相关课程。2.解决人工智能领域研究 所需的硬件条件, 保障专家学者 (包括外国专家) 富有竞 争力的物质保障, 为其工作 (现场或远程工作) 提供便利 条件。3.制定激励政策, 以科学发展和研发前景吸引企业 与个人为合作投资。4.加强两国之间具有优势学科的高校、 研究所之间的联系, 可派出学生学者进行长期访问与学习。 5. 在两国现有的研究基础上, 互派代表团进行交流与学习, 结合各自优势与特点建立中俄合作研发项目。6.支持两国 人工智能 (相关) 产品 (服务) 出口以及在世界市场上的 推广。

\section{6. 结语}

除以上所述, 针对众多人提出的问题和担忧: 人工智 能会不会取代人类, 人工智能教学是否会完全取代人类教 师? 答案是否定的, 现阶段, 人工智能教学系统或相关产 品作为人类研发出来的成果, 只能作为助手辅助教师完成 一些程序简单, 重复性的工作。未来可能发展到人机相互 合作甚至人类老师为人工智能产品做助手的程度来完成 教学。但学习不是一味的学习知识与技能, 还要再成长过 程中有情感的投入和有温度的交流, 这是人工智能产品不 具有的条件。简单来说, 人工智能产品可以优化教学, 但 永远不可能取代人类教师。

教育是个永恒的话题。人工智能未来的路还很长, 将 人工智能技术应用到教育领域中, 要正视其带来的便利和 不良后果, 避免盲目追赶潮流带来不可逆的负面影响。中 俄两国的合作要客观的看待所处的境况, 真诚沟通, 直面 问题, 取长补短, 合作共赢。让科技为人类所用, 以达到 国际先进水平。中俄双方在该领域的技术合作空间巨大, 是长期探讨的课题, 两国关系也会更加紧密, 携手发展。

\section{参考文献}

[1] 赵宏媚.普京: 俄罗斯要颁布人工智能技术战略[J].世界教育 信息,2019,32(14):76-77.

[2] 祝士明,刘帅瑶. 世界高校智能教育的发展脉络及启示 [J/OL].中国电化教育,2019(11):49-59. 
[3] 邱娜. 人工智能视阈下教育变革探究 [J]. 智库时 代,2019(43):84-85.

[4] 曾辉.人工智能技术在教育领域的应用 $[\mathrm{J}]$. 电子技术与软件 工程,2019(19):241-242.

[5] 张謷. “人工智能+教育”对高校教师教学的影响及应对策略 研究 $[\mathrm{A}]$. 中共沈阳市委、沈阳市人民政府、国际生产工

[6] 杨啸.关于大数据和人工智能的高校信息化服务 $[\mathrm{J}]$. 科技 风,2019(28):100.

[7] 王志华,杨世清.加大人工智能技术在中小学教育中应用探 析[J].计算机产品与流通,2019(10):272.

[8] Mukhamadieva K.B.教育中的人工智能: Gladyshkin A.O.年 轻专家眼中的创新建设[C]. (库尔斯克, 2014.12.5-6) 库尔 斯克, 2014.237-239.

[9] DORONKINA NADIIA, IVASIUK OKSANA. 人工智能系 统中的对话 [J].现代世界中的实际科学研究, 2019. № 3-4 (47): 54-59.
[10] Pereverzeva N.A., Mordvinova Zh.S. 人工智能技术在教育 领域推广的问题. 科学教育创新发展 [C].格罗德诺州立农 业大学，2018年: 142-144.

[11] Bessmertny. I.A.人工智能[M]. 圣彼得堡: 圣彼得堡国立大学 ITMO, 2010.132

[12] 关于杭州中学 “智慧课堂”的相关报道 [Z] http://www.sohu.com/a/232188256 198170

[13] 关于海风教育 $\mathrm{AI}$ 系统好望角的相关报道 [Z] : https://baijiahao.baidu.com/s?id=1598580283935115452\&wfr $=$ spider $\&$ for $=\mathrm{pc}$

[14] 人工智能对当前职业的影响[Z].新华网, 2018.09.19

[15] 大学、社会与人类未来.[R] 国际论坛.莫斯科2019.03.25. http://radio_mohovaya9.tilda.ws/rectors

[16] 普京签署俄罗斯联邦2030年前国家人工智能发展战略.[Z] 2019.10.N490 http://www.consultant.ru/cons/cgi/online.cgi?req $=$ doc\&base $=$ LAW\& $=335184 \&$ fld $=134 \&$ dst $=100015,0 \&$ rnd $=0.94489483$ 12770571\#040737065515225135 